\title{
On the Benefits of Optical Gain-Clamped Amplification in Optical Burst Switching Networks
}

\author{
M. Zannin, Student Member, IEEE, K. Ennser, Member, IEEE, S. Taccheo, D. Careglio, Member, IEEE, \\ J. Solé-Pareta, and J. Aracil, Senior Member, IEEE
}

\begin{abstract}
This paper investigates the performance of an all-optical method for amplification gain control to be applied in the next generation of optical networks. An erbium-doped fiber amplifier is implemented in a simple and passive all-optical configuration known as optical gain-clamped optical amplifier (OA). The paper investigates the dynamic performance of the $\mathrm{OA}$ and discusses the interplay of amplifier dynamics with different traffic statistics. The investigation concerns exhaustive characterization of bit error rate performances under typical optical burst switching (OBS) traffic as well as special case of sudden power variation at the amplifier input. All obtained results show a reduction in the amplifier output power overshot compared to the case where the same OA operates without any gain stabilization technique. As an example, in the typical OBS traffic scenario, a reduction of $3 \mathrm{~dB}$ is observed.
\end{abstract}

Index Terms-Burst traffic statistics, EDFA, optical amplifier gain stabilization, optical burst switching, optical gain clamping.

\section{INTRODUCTION}

I MPROVEMENTS on transmission capacity of optical networks and on broadband services have continuously increased the traffic to be processed on current point-to-point networks. The consequence is that complexity and data processing increase, as so does the cost. The latter is critical if the resources are not flexible and constant upgrades are required.

Several alternative techniques have been studied to make more efficient the use of the resources. One of the proposed solutions is the migration of switching technologies from the electronic to the optical domain, with information gathered in packets or bursts. Optical burst switching (OBS) [1] network is a proposed design whose goal is to carry data bursts transparently through the network as an optical signal. This allows different clients to use and share wavelength resources.

A characteristic of burst transmission is that the power of a single channel is constantly changing, depending on whether it

Manuscript received November 28, 2008; revised June 09, 2009. First published August 07, 2009; current version published October 16, 2009. This work was supported by the BONE-project ("Building the Future Optical Network in Europe"), a Network of Excellence funded by the European Commission through the 7th ICT-Framework Programme, and by the Welsh Assembly Government.

M. Zannin, K. Ennser, and S. Taccheo are with the Institute of Advanced Telecommunications, Swansea University, Swansea SA2 8PP, U.K. (e-mail: 397006@swansea.ac.uk; k.ennser@swansea.ac.uk; s.taccheo@swansea.ac.uk).

D. Careglio and J. Solé-Pareta are with Universitat Politècnica de Catalunya, Barcelona 08034, Spain (e-mail: careglio@ac.upc.edu; pareta@ac.upc.edu).

J. Aracil is with Universidad Autonoma de Madrid, Ciudad Universitaria de Cantoblanco, Madrid 28049, Spain (e-mail: javier.aracil@uam.es).

Digital Object Identifier 10.1109/JLT.2009.2029535 is transmitting or not. Considering a wavelength-division multiplexing (WDM) system with several burst channels, the total power oscillates according to the channels traffic at each instant.

Power variations of the OBS network may be a problem when considering amplifier dynamics. When an erbium-doped fiber amplifier (EDFA) operates in saturated regime, variations of the input power affect its gain. An increase of input power results in more stimulated emission, a phenomenon that reduces population inversion in the amplifier cavity, reducing, therefore, the gain of the amplifier. Analogously, a decrease of the input power of an amplifier in saturation reduces stimulated emission, increasing population inversion and the gain.

The influence of power variation on the EDFA gain depends, among other factors, on the burst interarrival time. While the amplifier is transparent to gigabit modulation rates, WDM burst channels randomly switched on and off impact on the amplifier gain. Gain changes translate into dynamic power fluctuations and degradation of the SNR in all channels, which accumulate with cascades of amplifiers [2]. The EDFA gain spectrum slope is also affected, meaning that different WDM channels will face different gains.

Stabilization of the amplifier gain is a possible solution to minimize bit error rate (BER) penalties. There are several techniques proposed based on electronics [3], [4] or optical control [5].

This article investigates the effectiveness of all-optical gainclamped amplification with typical OBS traffic. Main advantages are the use of passive components, low-cost, and easy to upgrade standard amplifiers. However, relaxation oscillation frequencies may accumulate spurious power variation along the amplifier chain [6]. Although these power variations are relatively small, so far no complete set of BER measurements were carried out relating the peak-to-peak oscillations to a BER value. In this paper, we report a thorough investigation of the optical gain-clamped optical amplifier (OGC-OA) dynamics behavior in terms of power evolution, eye diagrams, and BER.

The paper is organized as follows. Section II analyzes amplification impairments caused by power oscillations in OBS networks and investigates the effectiveness of an all-optical method for gain stabilization. Analyses performed are qualitative and quantitative, with measurement of the power fluctuations of the amplified signals, eye diagrams, and BER. Section III discusses the characteristics and statistics of burst traffic and describes a tailor-made OBS platform. The effectiveness of the clamping technique is experimentally analyzed with typical OBS traffic. Section IV concludes the paper. 


\section{EXPERIMENTAL INVESTIGATION OF BURST AMPLIFICATION}

\section{A. Experiment Setup}

The setup implemented for the experiments described on this article represents a scenario of burst traffic with 16 WDM channels, which is optically amplified. The experiments focus on the degradation in transmission caused by the variation of power at the amplifier input. The constant adding and dropping of channels is perceived by the amplifier as a total input power variation, regardless of which channels are added or dropped. This leads to the assumption that the traffic can be represented by a single laser source, instead of 16 , provided that the traffic behavior is known. The laser source here contains the traffic total power concentrated at a single wavelength. This is actually a worse scenario than distributing the power into several channels spread over different wavelengths across the C-band, as it happens in a real system. In fact in the latter case, the negative effect of spectral hole burning (SHB) [7] is reduced.

The traffic is simulated here with a tunable laser set to -1 $\mathrm{dBm}$ and $1550 \mathrm{~nm}$. The signal is modulated according to the pulse pattern generator (PPG2) connected to the acousto-optic modulator (AOM). The PPG2 provides the AOM with the number of channels being transmitted simultaneously over time. This means that the power level of the tunable laser varies with the number of channels being transmitted.

Signal amplification is achieved with a standard commercial EDFA whose pump power is set to $155 \mathrm{~mW}$, which corresponds to a $17 \mathrm{~dB}$ gain with $-1 \mathrm{dBm}$ input power. In addition, the amplifier is also used as active medium in a laser cavity resonator configuration [5], which stabilizes its gain by providing optical feedback. Cavity losses are determined by a variable attenuator (VA), coupling losses of the ring, and the insertion losses of the optical devices used. For sake of simplicity, the ring cavity configuration used allows the lasing wavelength to be varied with a tunable narrowband filter $(0.2 \mathrm{~nm}$ full-width at half-maximum) present in the cavity loop. During the experiment, the tunable narrowband filter was centered at $1548 \mathrm{~nm}$, which determines the resonating wavelength. This choice reduces the SHB effect due to the use of a single 16-channel source, with the laser and signal wavelengths quite close to each other. [8]. To guarantee the clamping robustness, the pump power is increased by a factor $x=1.30$ with respect to the pump power needed to start lasing action. The pump power is defined as $P_{\text {Pump }}=x \cdot P_{\text {Pump,th }}$, where $P_{\text {Pump,th }}$ is the pump power at the lasing threshold, and it is about the same power needed to drive the OA to the same gain level without clamping. Note that the cavity passive length is minimized (around $5 \mathrm{~m}$ ) in order to enhance the performance of the clamping method [9].

In a laser system, there are intrinsic dynamics related to the relaxation oscillations-i.e., the natural frequency at which the laser power (hence the amplifier gain) oscillates once the system is perturbed. These gain oscillations may impact the quality of the signal amplified; therefore, an investigation is carried out.

In order to test the signal quality, the experiment uses a probe channel with average power of $-15 \mathrm{dBm}$ at $1556.5 \mathrm{~nm}$. The probe channel is configured either as a continuous wave or as a binary sequence, depending on the experiment. In the latter,

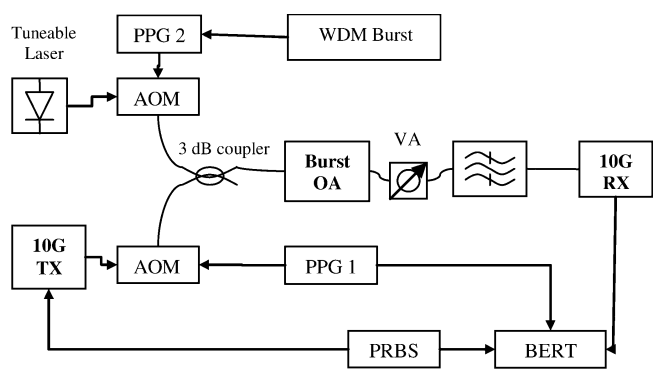

Fig. 1. Experimental setup for WDM burst signal amplification with gain-clamping configuration and BER measurement.

a pseudorandom binary sequence generator (PRBS) provides a Mach-Zehnder modulator with a $2^{31}-1$ sequence at $10 \mathrm{Gbit} / \mathrm{s}$. After this transmitter block (10G TX), an AOM acts as a switch for simulating burst transmission with pattern determined by the PPG1.

A BER tester (BERT) compares the data generated with the one identified by the receiving block (10G RX). The optical bandpass filter (OBPF) is tuned to the probe channel wavelength while the power received is controlled by a VA.

\section{B. On-Off Cases}

The first set of experiments is devoted to evaluate the gain excursion induced by large and abrupt (less than $1 \mu$ s rise/fall time) change in the input power. The first approach simulates a worst-case scenario, where the maximum variation in power occurs. This represents the case where the system undergoes sudden drop of all the channels transmitted and, analogously, all the channels are simultaneously switched on, due to channel reconfiguration.

The tunable laser that represents all the channels is modulated by a square wave at $1 \mathrm{kHz}$, with maximum power equal to -1 $\mathrm{dBm}$. The value is set higher on this experiment in order to stress the worst case of operation of the EDFA, which guarantees that it operates in the saturation regime.

The gain variation of the surviving channel represented by the probe channel is analyzed both with and without gain clamping. The gain excursion of the probe channel is shown in Fig. 2. We note an offset of nearly $6 \mathrm{~dB}$ for unclamped condition while the power excursion is limited to $0.5 \mathrm{~dB}$ when the OGC-OA configuration is used. Expanded details of OGC-OA measurement are shown in Fig. 3.

The experiment is repeated with lower signal power variation. The maximum power is now set to $-7 \mathrm{dBm}$, which represents a 4-channel WDM system. Fig. 4 shows the gain variation for the OA without and with gain stabilization. The offset for the first case is around $3.1 \mathrm{~dB}$, while clamping the gain limits the power excursions to less than $0.2 \mathrm{~dB}$ (shown in detail on Fig. 5).

Both cases showcase significant reduction in the maximum gain excursion when OGC-OA is used. Also note that even the worst case-16-channel ON/OFFshown in Fig. 3-indicates that the clamped gain amplifier reduces considerably the ripple to a value of $0.5 \mathrm{~dB}$, which is quite reasonable.

A second set of experiments evaluates the impact of gain excursion on eye-diagram quality and BER measurements. For this purpose, the continuous probe channel is replaced by a 


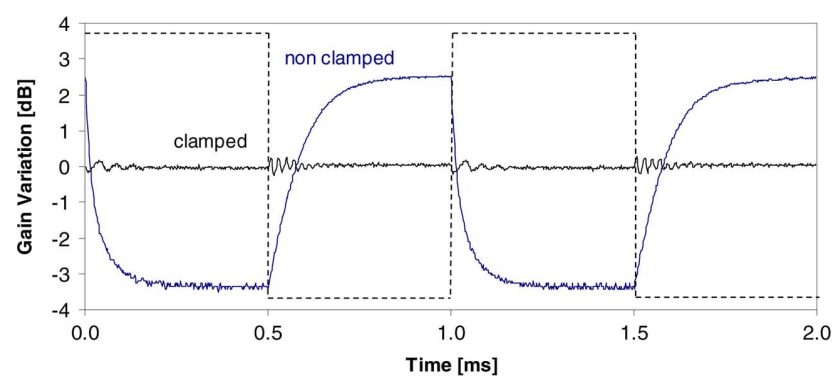

Fig. 2. Gain variation of the surviving channel due to simultaneous drop and addition of 16 channels at $1 \mathrm{kHz}$ on a WDM system with both clamped and nonclamped optical amplification.

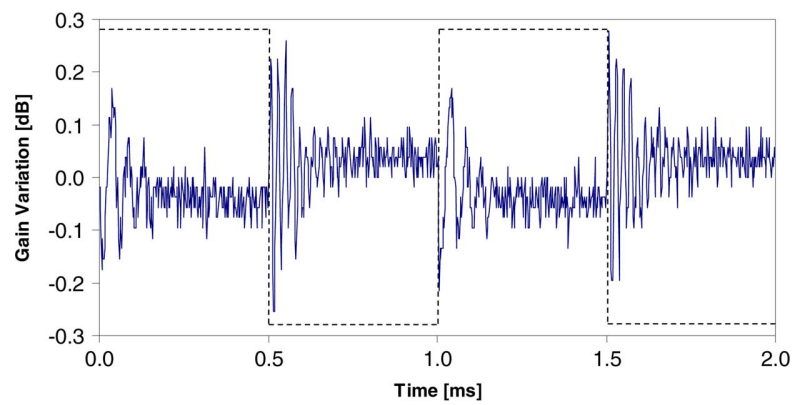

Fig. 3. Expanded details of Fig. 2 curve representing probe channel gain variation with optically clamped gain.

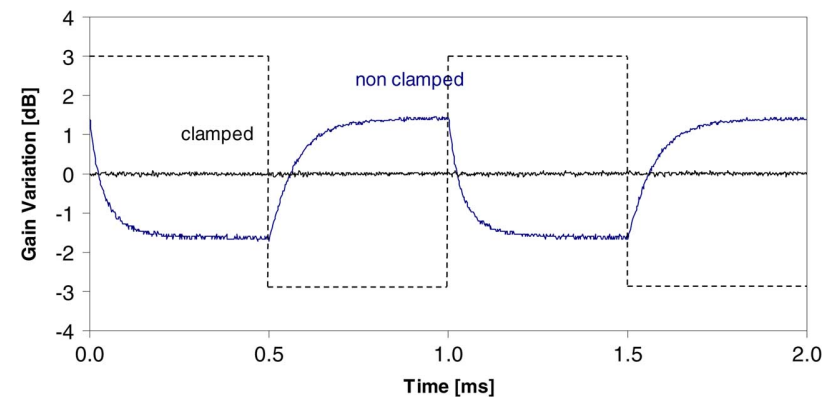

Fig. 4. Gain variation of the surviving channel due to simultaneous drop and addition of 4 channels on a WDM system with both clamped and nonclamped optical amplification.

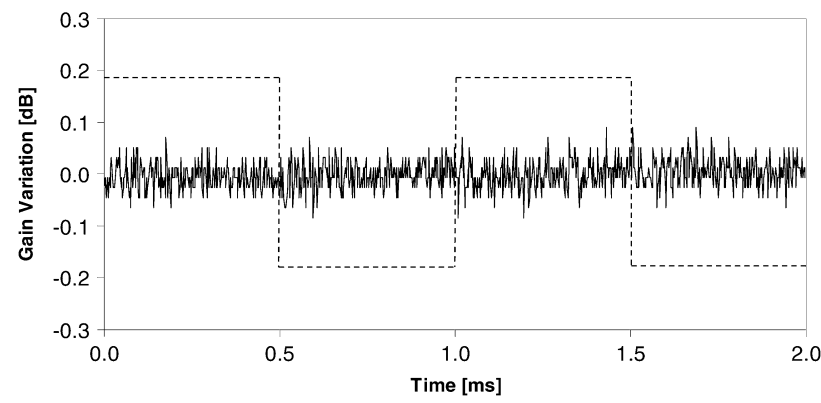

Fig. 5. Gain variation of the surviving channel due to simultaneous drop and addition of 4 channels at $1 \mathrm{kHz}$ on a WDM system with optically clamped gain.

$10 \mathrm{Gbit} / \mathrm{s}$ PRBS, as described on Section II-A. Fig. 6 shows the 16-channel ON-OFFscenario: (a) with the nonclamped and (b) the clamped gain amplifier. The scenario representing ON-OFFof 4 channels is shown in Fig. 7: (a) nonclamped EDFA and (b) clamped gain amplifier.
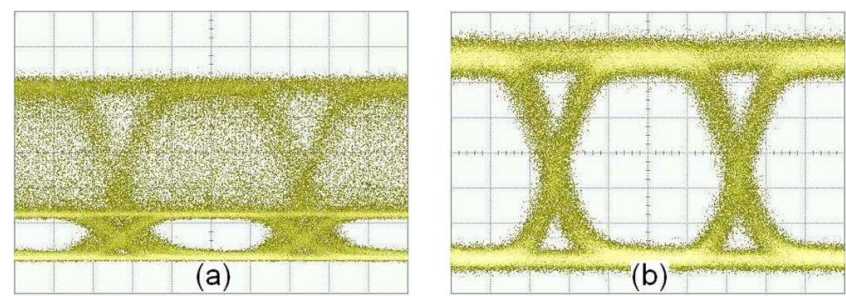

Fig. 6. Eye diagram of the probe channel under simultaneous drop and addition of 16 channels on a WDM system with both (a) nonclamped and (b) clamped optical amplification.
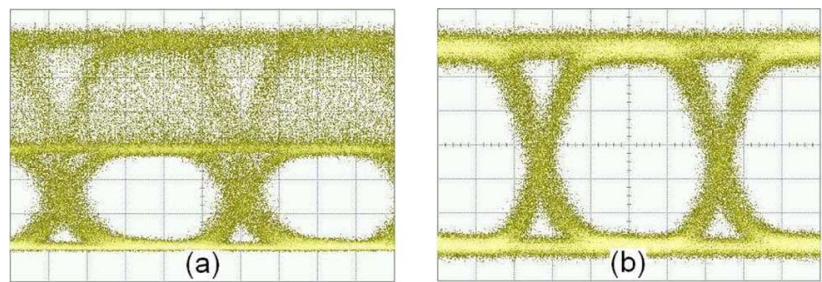

Fig. 7. Eye diagram of the probe channel under simultaneous drop and addition (ON/OFF) of 4 channels on a WDM system with (a) nonclamped and (b) clamped optical amplification.

Fig. 6(a) shows a noisy eye diagram with three level signals. The lower level corresponds to the transmission of logic level 0s. The intermediate level corresponds to logic level 1s when all channels are present (simultaneously transmitted). The higher level corresponds to logic level $1 \mathrm{~s}$ when the probe channel is the only channel transmitted.

If the input power of the amplifier is reduced, the amplifier operation is closer to linear regime, which increases its gain. This is the reason that the eye opening height is higher in Fig. 7(a) than in Fig. 6(a). Note that the two figures are in the same scale.

To quantify the evidence of eye diagram differences, we measured BER values as shown in Fig. 8. The BER curves of the two clamped cases are similar. In fact, comparing once again the cases with 16 and 4 channels with clamped gain, the slight difference in ripples shown in Figs. 3 and 5 is not noticeable in the eye diagrams of Figs. 6(b) and 7(b). If instead the BER curve comparison is made among the unclamped cases, the penalty induced with large number of channel is higher, more than $1.5 \mathrm{~dB}$ at $\mathrm{BER}=10^{-9}$, which is in accordance to the difference observed in the eye diagrams in Figs. 6(a) and 7(a).

The curves demonstrate that the OGC-OA is robust in terms of BER for both $\mathrm{ON}-\mathrm{OFF}$ cases at a $1-\mathrm{kHz}$ rate, with $3-\mathrm{dB}$ improvement for the worst case (16 channels). Since these simultaneous addition and drop of a large channel number is unlikely to occur, the results indicate that input power variation at this given rate do not affect the robustness of the clamping configuration.

Further understanding of the behavior of the amplifier is obtained by varying the rate of the input power steps. In fact, as previously mentioned, the OGC-OA has some intrinsic dynamics, related to the relaxation oscillation frequency (ROF), while the unclamped OA has a rise/fall dynamic with time constant related to erbium $I_{13 / 2}$ lifetime of about $8 \mathrm{~ms}$ in silica fibers. The worst case of 16 channels ON-OFFis implemented and the channels add/drop frequency is varied. 


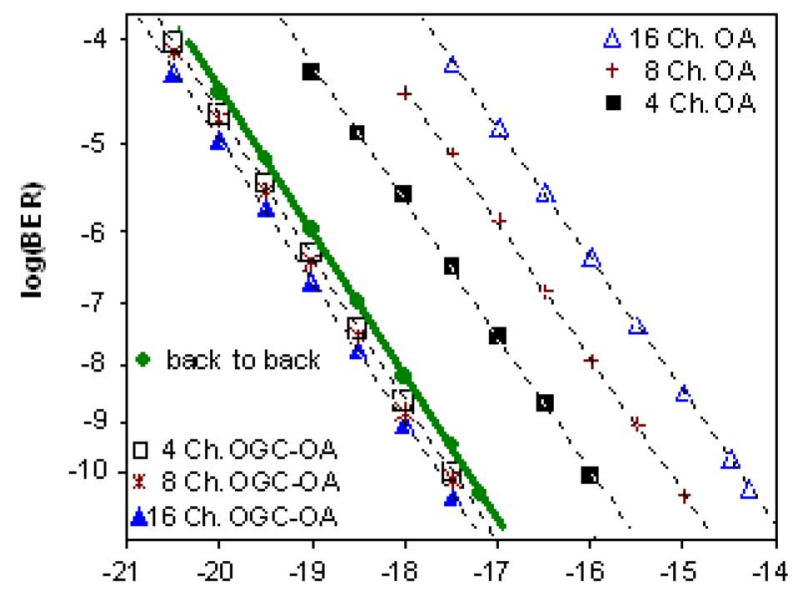

Fig. 8. BER curves for the probe channel under simultaneous drop and addition of 16,8 , and 4 channels at $1 \mathrm{kHz}$ on a WDM system with both clamped and nonclamped optical amplification.

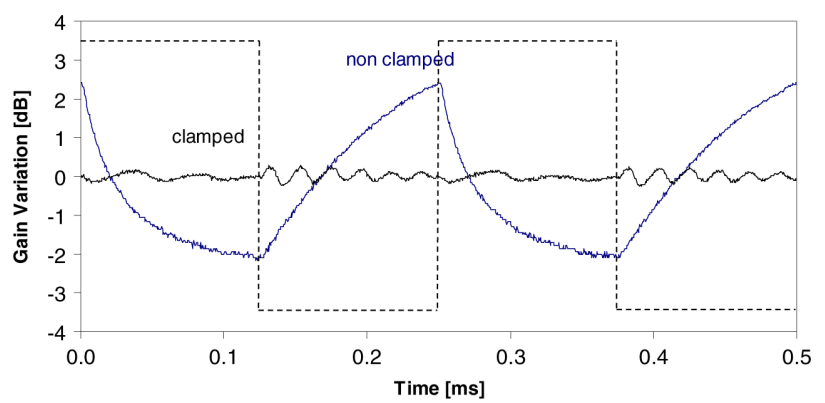

Fig. 9. Gain variation of the surviving channel (probe) due to simultaneous drop and addition (ON/OFF) of 16 channels at $4 \mathrm{kHz}$ on a WDM system with clamped and nonclamped optical amplification.

An increase of the ON-OFF frequency has nontrivial meaning: for OGC-OA it implies that the OA has less time for stabilizing its gain but also that the perturbation lasts less time; for unclamped OA it implies less time for gain rising and when the frequency is faster than the erbium lifetime, an equivalent average input power is seen by the unclamped amplifier. Fig. 9 shows the case where the frequency is $4 \mathrm{kHz}$. This gain variation should be compared with Fig. 2, where the frequency is $1 \mathrm{kHz}$ for the same power. For the case where the gain is not clamped, it is possible to observe that the faster change of input power does not allow enough time for the gain to stabilize.

Increasing the frequency to $10 \mathrm{kHz}$, the effect is even clearer, as shown in Fig. 10. The response of the amplifier is slow if compared to the $\mathrm{ON}-\mathrm{OFF}$ frequency and this may generate interplay between oscillations in the ON and OFFtime slots [10].

Fig. 11 shows that at a frequency equal to $100 \mathrm{kHz}$, the amplifier is nearly insensitive to the changes in the input power of the amplifier. Therefore, there is no significant difference in clamping or not the amplifier gain.

By comparing the cases with clamped gain in Figs. 2, 9, and 10 , one observes that the initial ripples behave the same way-apart from an interplay behavior that is observed in Fig. 10 because the add/drop frequency approaches the resonator cavity ROF, which could induce chaotic behavior as reported in [10]. Nevertheless, these ripples are not enough to

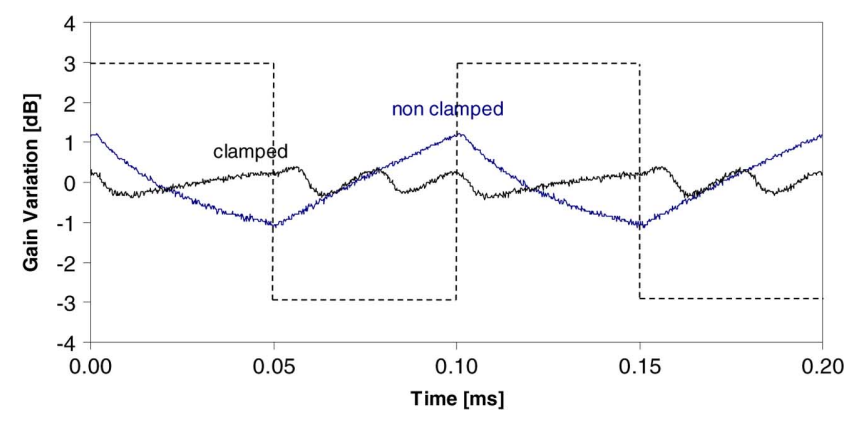

Fig. 10. Gain variation of the surviving channel (probe) due to simultaneous drop and addition (ON/OFF) of 16 channels at $10 \mathrm{kHz}$ on a WDM system with clamped and nonclamped optical amplification.

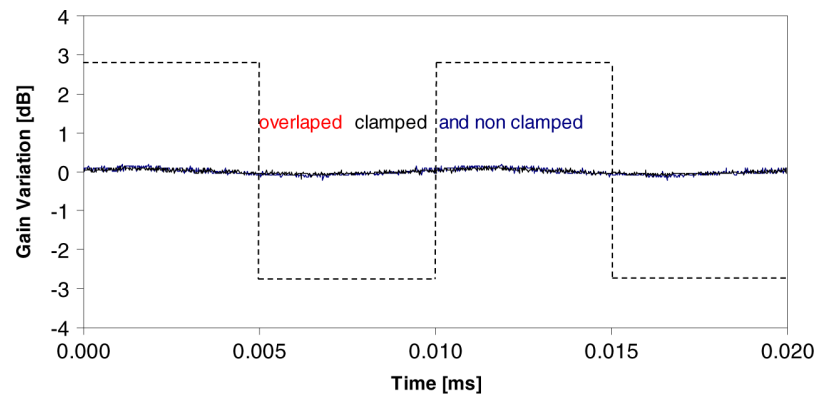

Fig. 11. Gain variation of the surviving channel due to simultaneous drop and addition of 16 channels at $100 \mathrm{kHz}$ on a WDM system with both clamped and nonclamped optical amplification.

affect the BER. This may be observed in Fig. 12, where the cases with clamped gain are roughly equal within uncertainties. On the contrary, as expected, gain ripples in Figs. 2, 9-11 decrease for unclamped OA. Therefore, unclamped OA looks more stable for very fast sustained modulations in the range of $10 \mathrm{kHz}$ to about $100 \mathrm{kHz}$. However, we note here that, as discussed about the OBS traffic, it is very unlikely to have more than two ON/OFF modulation at the same frequencies. Therefore, the ripples of OGC-OA, originated by long sequences of ON/OFF modulation, are likely to be much smaller with real traffic [11]. This constitutes the investigation reported in the next section of this paper.

\section{Characteristics of Burst Traffic}

An OBS network consists of a set of edge and core nodes connected by WDM links. At the edge nodes, packets coming from the client networks are assembled according to their forwarding equivalence class into so-called bursts (burstification process). After transmission through the network toward their destination, the bursts are disassembled at the egress and the packets are forwarded to the destination clients. The main design objective is that the bursts are carried transparently inside the OBS network as an optical signal. Besides, the control information is carried on a dedicated channel separately from the burst. In such a network, the wavelength resources are temporarily utilized and shared between different clients. The aggregation of user data (i.e., the bursts) helps to improve the network scalability, as well as it relaxes the switching requirements [1]. 


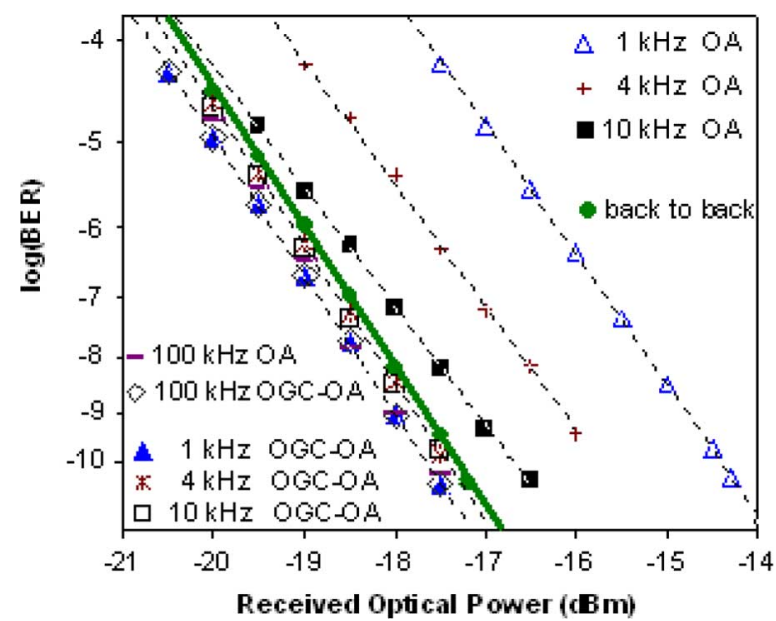

Fig. 12. BER curves for the probe channel under simultaneous drop and addition of 16 channels at rates of $1,4,10$, and $100 \mathrm{kHz}$ on a WDM system with both clamped and nonclamped optical amplification.

Prior to the evaluation of the impact of OBS traffic in OGC-OA/OA scenarios, we first provide a description of the traffic statistics, together with the burstification process, which is the main response of such statistics [12]. In the following discussions, we briefly present the different burstifiers proposed in OBS and its impacts on both commonly considered traffic models and real data collected in a metro-regional link.

\section{A. Burstification Process}

The burstifier (also known as burst assembly) installed in the edge nodes is in charge of producing the optical bursts. Burstification algorithms can be classified as timer-based, size-based, and hybrid timer/size-based [13]. In the timer-based scheme, a timer starts upon the arrival of the first packet to an empty queue, i.e., at the beginning of a new assembly cycle. After a fixed time, all the packets arrived in this period are assembled into a burst. In the size-based scheme, a burst is sent out when enough packets have been collected in the assembly queue such that the size of the resulting burst exceeds a given threshold. In the hybrid algorithms, a burst can be sent out when either the burst length exceeds the desirable size or the timer expires.

Recently some threshold-adaptive, hybrid burstifiers are proposed to eventually respond to traffic changes. They are not considered here since its impact on the traffic statistics cannot be easily determined.

\section{B. Analytical Models}

The statistical properties of the traffic are highly dependent on the burstification algorithm. Indeed, packets coming from the client networks have to wait in the electronic aggregation queues some amount of time until the given thresholds are reached. Depending on the threshold (size, time, or hybrid), the burst interarrival times are different.

On the other hand, the statistical features of the outgoing traffic from the burstifier also depend on the input traffic. Let us make a distinction between Poisson, self-similar, and multifractal input in the following sections.
1) Poisson Input: For size-based burstifiers, the burst size is constant and the interarrival times follow an Erlang distribution, as pointed out in [14]. Actually, the burst interarrival time can be modeled by a (finite) sum of independent exponential random variables, which gives raise to an Erlang distribution. On the other hand, timer-based burstifiers provide constant interarrival times and a burst size, which converges in distribution to Gaussian. Note that the timer enforces a constant interarrival time, whereas the burst size distribution can be explained by means of the Central Limit Theorem. Actually, bursts are made up of a random number of packets, which arrive in between consecutive timeouts. As a result, the burst size is a sum of independent random variables. If the link speed is high, a large number of packets are expected per burst, and the Gaussian distribution follows. Finally, the analytical expressions for burst size and interarrival times for hybrid burstifiers are rather involved and they largely depend on the load conditions. If the load is high, chances are that the size-based threshold expires continuously, and the traffic resembles that of a size-based burstifier. Conversely, in low load situations, the timer is likely to expire, and the generated traffic is similar to that of a timer-based burstifier.

2) Self-Similar Input: In the self-similar case, the input traffic is a continuous-time Gaussian stochastic process with heavytailed auto-correlation, namely the auto-correlation presents a hyperbolic decay with the lag. The analysis presented in [15] shows that timer-based burstifiers provide a Gaussian burst-size, because the input is a Gaussian fluid, and constant-interarrival times. Furthermore, size-based burstifiers show nearly Gaussian interarrival times and constant burst size. Hybrid burstifiers are somehow in between; several burstification strategies are considered in [16] where an analysis of the burstifier outgoing traffic based on different combinations of size and time is provided.

3) Multifractal Input: A more complex version of self-similarity is the multifractal traffic model [17]. As self-similar, this model shows a slowly decaying dependence structure. The difference is that the scaling property of this process is not uniform across multiple time scales and orders of statistics. Currently, there are no investigations on the burst statistics when multifractal model is considered as input traffic.

\section{Real Data Collection and Traffic Characterization}

We consider a scenario where several client networks are attached to a single OBS node, which performs packets aggregation and bursts generation. For the client traffic, we use real packet traces captured with an ad hoc measurement platform designed to operate at gigabit speed with no packet loss and nanoseconds precision in the packet time stamp [18]. The point of measurement is a pair of Full-Duplex Gigabit Ethernet links (two per each traffic direction, thus $2 \mathrm{Gbit} / \mathrm{s}$ per direction) that connects the Catalan R\&D network (about 50 Universities and Research Centers) with the Spanish R\&D RedIris network and to the global Internet. The importance of having nanoseconds precision is fundamental to capture the correct statistic properties of the traces and we note that worse accuracy produces completely different results [19]. In this metro-regional link, the monitored packet traces do not present Poisson or monofractal 


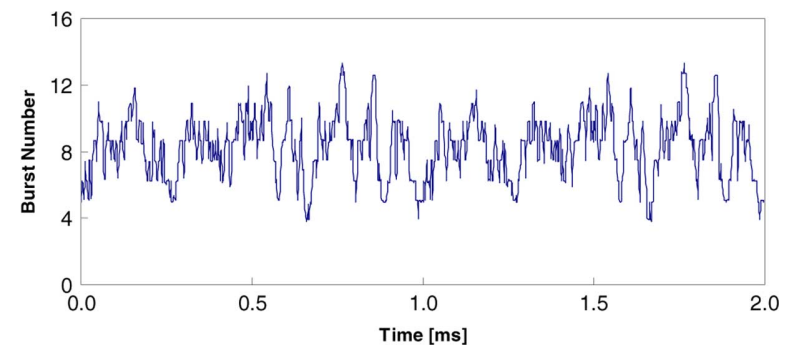

Fig. 13. Optical burst data at the amplifier input.

self-similar properties but complex multifractal scaling behaviors [19]. Thus, we perform our evaluation with these real traces.

At the OBS edge node, a hybrid timer/size threshold burstifier is used to aggregate the packets. To emulate the aggregation of different client networks, several real traces have been multiplexed in order to have 0.5 load on average in a $10 \mathrm{Gbit} / \mathrm{s}$ channel. The timer threshold of the burstifier is set to $5 \mathrm{~ms}$ and the maximum burst length to 250 Kbytes. To stress the EDFA, a guard time of $10 \mathrm{~ns}$ is applied to separate the bursts. We assume that the OBS network domain is composed of 30 nodes; the destination of the bursts is obtained aggregating the IP addresses of the packet traces according to their geographical location.

As commented in Section III-B, the obtained burst trace changes the short-range characteristics of the packet trace and has quasi-Gaussian distributed both interarrival time and burst size with bounded marginal distributions. On the contrary, the long-range characteristics maintain the same statistics as the packet traces, i.e., they have nontrivial multifractal scaling behavior.

\section{Experimental Analyses With Typical Burst Traffic}

The power variation on the input of the amplifier is now determined by the burst traffic data discussed in the previous section. The ad hoc measurement platform described on Section III-C provides 14-s traces composed by 95000 bursts. A 1-s long WDM burst stream is assembled from uncorrelated data samples from different time slots. Burst lengths are in the order of tens of microseconds. A sample of the burst optical signal is shown in Fig. 13 with the power scaled to the number of channels. The average power at the input of the amplifier is $-4 \mathrm{dBm}$ and corresponds to the level of 8 channels.

The gain variation for the both clamped and nonclamped amplifier is shown in Fig. 14. The $3.3 \mathrm{~dB}$ ripples that are present when the nonclamped amplifier is used are within $0.3 \mathrm{~dB}$ for the clamped case.

The eye diagram of the nonclamped amplified burst traffic data in Fig. 15(a) is less noisy than the cases discussed in the previous section: Figs. 6(a) and 7(a). This phenomenon is observed in the case of burst traffic because the power variation steps are smaller than the ones simulated in the previous cases. Another factor that contributes to this improvement is the fact that the steps occur at a faster rate than the one in the previous eye diagrams.

Fig. 16 shows that with OCG-OA, the performance of the system is improved by $1.5 \mathrm{~dB}$ at a BER equal to $10^{-10}$.

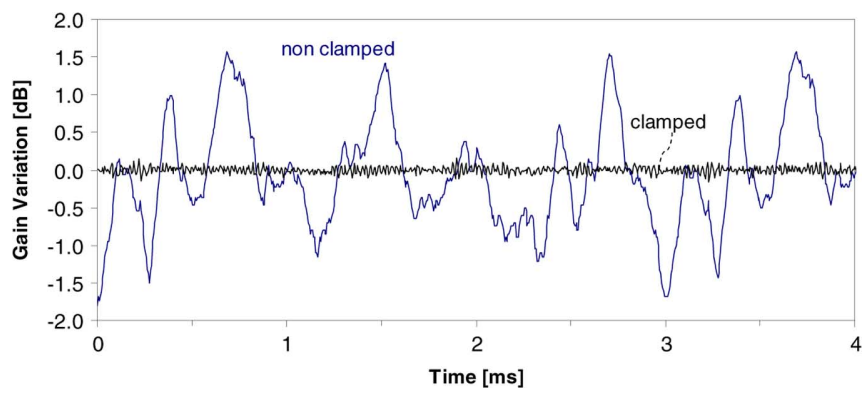

Fig. 14. Gain variation of the surviving channel in a 16-channel WDM system with burst traffic with both clamped and nonclamped optical amplification.

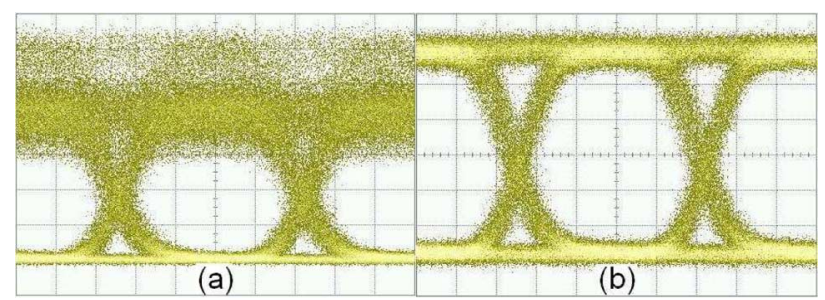

Fig. 15. Eye diagram of the probe channel in a 16-channel WDM system with burst traffic with (a) nonclamped and (b) clamped optical amplification.

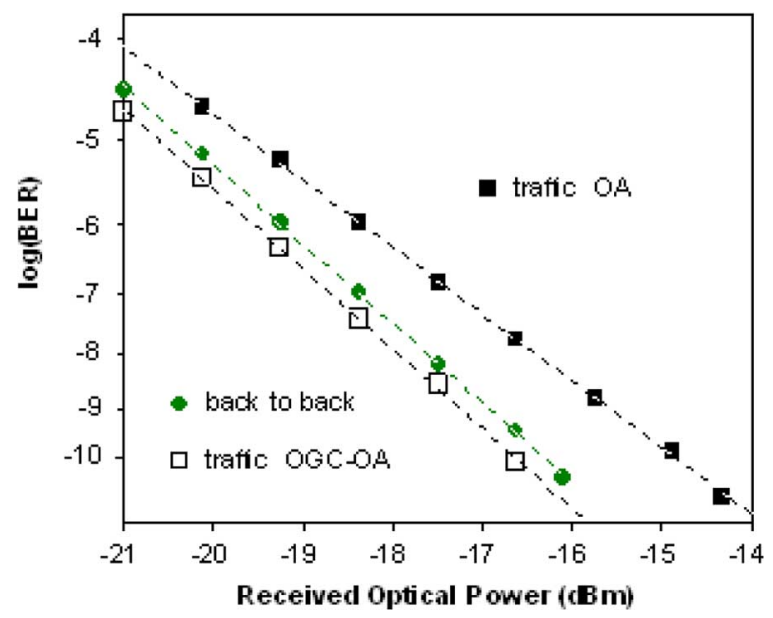

Fig. 16. BER curves for the probe channel in a 16-channel WDM system with burst traffic with both clamped and nonclamped optical amplification.

\section{CONCLUSION}

The effectiveness of optical gain-clamping technique to stabilize amplifier at the presence of OBS traffic is investigated. Abrupt $1 \mathrm{kHz}$ add/drop of 16 channels result in power excursions of $6 \mathrm{~dB}$ for the surviving channel when no gain stabilization technique is implemented. The passive all-optical clamping method limits power excursions to $0.5 \mathrm{~dB}$. The gain control reduces transmission penalty by $3 \mathrm{~dB}$ when $\mathrm{BER}=10^{-10}$. Cutting the number of channels from 16 to 4 reduces the nonclamped power excursions by $3 \mathrm{~dB}$, which translates into $1.5 \mathrm{~dB}$ improvement for $\mathrm{BER}=10^{-10}$. Applying the clamping techniques still improves the BER by additional $1.5 \mathrm{~dB}$ while power excursions are limited to $0.2 \mathrm{~dB}$. The $0.3 \mathrm{~dB}$ difference between clamped amplifiers with 16 and 4 channels are not sensed by the system in terms of performance: the BER curves are similar. 
When typical OBS traffic is used, the average input power remains the same, corresponding to 8 channels. Nevertheless, the variation steps are smaller and therefore the nonstabilized gain amplifier causes the output power to fluctuate within $3.3 \mathrm{~dB}$. Applying the clamping technique described here limits these power excursions to $0.3 \mathrm{~dB}$. This $3 \mathrm{~dB}$ improvement increases the system performance by $1.5 \mathrm{~dB}$ with $\mathrm{BER}=10^{-10}$.

\section{REFERENCES}

[1] C. Qiao and M. Yoo, "Optical burst switching (OBS) - A new paradigm for an optical internet," J. High Speed Netw., vol. 8, no. 1, pp. 69-84, 1999.

[2] J. L. Zyskind, Y. Sun, A. K. Srivastava, J. W. Sulhoff, A. J. Lucero, C. Wolf, and R. W. Tkach, "Fast power transients in optically amplified multiwavelength optical networks," in Proc. OFC 1996, USA, , paper PD31.

[3] A. K. Srivastava, Y. Sun, J. L. Zyskind, J. W. Sulhoff, C. Wolf, and R. W. Tkach, "Fast gain control in an erbium-doped fiber amplifier," in Proc. Opt. Amplifier Appl., 1996, pp. 1-3, paper PD-P4.

[4] N. Madamopoulos, D. C. Friedman, I. Tomkos, and A. Boskovic, "Study of the performance of a transparent and reconfigurable metropolitan area network," J. Lightw. Technol., vol. 20, no. 6, pp. 937-945, Jan. 2002.

[5] M. Zirngibl, "Gain control in erbium-doped fibre amplifiers by an alloptical feedback loop," Electron. Lett., vol. 27, no. 7, pp. 560-561, 1991.

[6] K. Ennser, S. Taccheo, T. Rogowski, and J. Shmulovich, "Optical stabilization of waveguide amplifiers for WDM ring network with recirculating optical power," J. Lightw. Technol., vol. 25, no. 7, pp. 1670-1675, Jul. 2007.

[7] G. Luo, J. L. Zyskind, J. A. Nagel, and M. A. Ali, "Experimental and theoretical analysis of relaxation-oscillation and spectral hole burning effects in all-optical gain-clamped EDFA's for WDM networks," $J$. Lightw. Technol., vol. 16, no. 4, pp. 527-533, Apr. 1998.

[8] K. Ennser, S. Taccheo, T. Rogowski, and J. Shmulovich, "Efficient erbium-doped waveguide amplifier insensitive to power fluctuations," Opt. Exp., vol. 14, pp. 10307-10312, 2006.

[9] K. Ennser, G. della Valle, M. Ibsen, J. Shmulovich, and S. Taccheo, "Erbium-doped waveguide amplifier for reconfigurable WDM metro networks," IEEE Photon. Technol. Lett., vol. 17, no. 7, pp. 1468-1470, Jul. 2005.

[10] G. Della Valle, A. Festa, S. Taccheo, K. Ennser, and J. Aracil, "Nonlinear dynamics induced by burst amplification in optically gain-stabilized erbium-doped amplifiers," Opt. Lett., vol. 32, no. 8, pp. 903-905, 2007.

[11] S. Taccheo, G. Della Valle, A. Festa, K. Ennser, and J. Aracil, "Amplification of optical bursts in gain-stabilized erbium-doped optical amplifier," in Proc. Opt. Fiber Commun. Conf. (OFC 2007), , USA, , pp. $1-3$, paper OMN3.

[12] X. Yu, J. Li, X. Cao, Y. Chen, and C. Qiao, "Traffic statistics and performance evaluation in optical burst switched networks," J. Lightw. Technol., vol. 22, no. 12, pp. 2722-2738, Dec. 2004.

[13] X. Yu, Y. Chen, and C. Qiao, "Study of traffic statistics of assembled burst traffic in optical burst switched networks," in Proc. Opticomm, 2002, pp. 149-59.

[14] J. Hernandez, J. Aracil, V. Lopez, and J. Lopez de Vergara, "On the analysis of burst-assembly delay in OBS networks and applications in delay-based service differentiation," Photon. Netw. Commun., vol. 14, no. 1, pp. 49-62, 2007.

[15] M. Izal and J. Aracil, "On the influence of self similarity on optical burst switching traffic," in Proc. Globecom 2002, Taipei, China, Nov. 2002, pp. 2308-2312.

[16] M. Izal, J. Aracil, D. Morató, and E. Magaña, "Delay-throughput curves for timer-based obs burstifiers with light load," J. Lightw. Technol., vol. 24, no. 1, pp. 277-285, Jan. 2006.

[17] D. Veitch, N. Hohn, and P. Abry, "Multifractality in TCP/IP traffic: The case against," Comput. Netw., vol. 48, no. 3, pp. 293-313, 2005.

[18] P. Barlet, J. Sole-Pareta, J. Barrantes, E. Codina, and J. DomingoPascual, "SMARTxAC: A passive monitoring and analysis system for high-speed networks," in Proc. Terena Netw. Conf., Catania, Italy, May 2006, pp. 1-10.

[19] M. de Vega, S. Spadaro, M.-A. Remiche, D. Careglio, J. Barrantes, and J. Götz, "On the statistical nature of highly-aggregated internet traffic," in Proc. Int. Workshop Internet Perf., Simul., Monitoring Meas., Salzburg, Austria, Feb. 2006, pp. 29-39.
Marcelo Zannin (S'09) obtained the Graduate degree in electrical engineering from the University Federal of Santa Catarina, Santa Catarina, Brazil, in 2005, the Master's degree in optical communications and photonic technologies at Politecnico di Torino, Torino, Italy, in 2006. He is currently working toward the $\mathrm{Ph} . \mathrm{D}$. degree in telecommunications engineering at the Institute of Advanced Telecommunications, Swansea University, Swansea, U.K.

During his undergraduate degree, he was a Research Student at the Laboratory of Digital Signal Processing, University Federal of Santa Catarina. During his Master's degree, he was a Research Assistant at Optcom, Politecnico di Torino, working on electronic dispersion compensation for optical communications. $\mathrm{He}$ has been involved in the European project e-photon 1 and is now involved in BONE, both European projects. His current research interests include optical amplifier gain control.

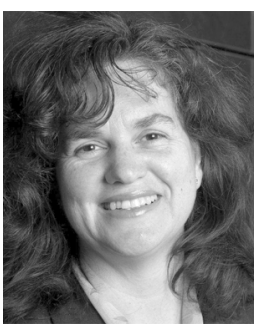

Karin Ennser (M'98) received the B.S. and M.S. degrees in electrical engineering from the State University of Campinas, São Paulo, Brazil, in 1990 and 1993, respectively, and the Ph.D. degree in electrical engineering from the Technical University of Berlin, Berlin, Germany, in 1998.

In 1996, she was a Visiting Researcher at Optoelectronic Research Center, University of Southampton, where she studied and designed chirped fiber Bragg gratings for telecom applications. From 1998 to middle 2000, she joined Corning Inc., as a Senior Research Scientist working on devices and system aspects of DWDM networks. From middle 2000 to beginning of 2003, she worked in Pirelli Telecom Cavi e Sistemi S.p.A., as a Product Development Manager of telecom optical fiber. From 2003 to 2006, she joined the National Laboratory of Photonic Networks of Italian Inter-University Consortium on Telecommunications, Italy, as the Head of Research in the area of optical components and system applications. In 2006, she joined the Institute of Advanced Telecommunications of Swansea University, Swansea, U.K. She has authored or coauthored more than 80 publications in international journals and conferences and holds several patents. His recent research interests include transient gain dynamics in doped fiber and waveguide amplifiers, all-optical signal processing, transmission system design, and performance-related issues.

Dr. Ennser acts as a Reviewer of major journals and letters and as member of technical committees of international conferences.

Stefano Taccheo received the M.S. degree in nuclear engineering, in 1989, from Politecnico di Milano, Milano, Italy, and the Ph.D. degree in applied physics from Politecnico di Turin, Italy, in 1996.

From 1990 to 1991, he worked as a Researcher at CSELT Turin, Italy. From 1991 to 1996, he was a Researcher at the Centre of Quantum Electronic of the National Research Council, Milano. In 1997, he was a Visiting Researcher at the Optoelectronic Research Centre, Southampton. He was appointed as Assistant Professor and Associate Professor at the Physics Department, Politecnico di Milano, Italy, in 1998 and 2004, respectively. In 2007, he joined the Institute of Advanced Communications of Swansea University, Swansea, U.K. In 1998, he spent a Sabbatical year at Lightwave Transmission Group at Lucent Bell-Labs. He has authored and coauthored more than 200 journal and conference publications. He has been a consultant of several laser and Telecom companies, and he has been an appointed Chair of several conferences and workshops of the IEEE and the European Optical Society. His research interests include fiber lasers, new glasses as active materials, solid-state laser, optical amplifiers, continuum sources, and optical components. Target applications are mainly sensing, security\&defense, biomedicine, and optical communications.

Davide Careglio (S'05-M'06) received the M.Sc. and Ph.D. degrees in telecommunications engineering from the Universitat Politècnica de Catalunya (UPC), Barcelona, Spain, in 2000 and 2005, respectively, and the Dr.Ing. degree in electrical engineering from Politecnico di Torino, Torino, Italy, in 2001.

Currently, he is an Associate Professor at the Department of Computer Architecture, UPC. Recently, he has been involved in several European Projects. He is currently participating in the FP7 Strep Project DICONET and in the FP7 Network of Excellence BONE. His research interests include all-optical networks with emphasis on packet-based switching technologies, Quality of Service $(\mathrm{QoS})$ provisioning, and traffic engineering. 
Dr. Careglio has participated in the technical program committees of several conferences, including IEEE ICC and IEEE Globecom. He is a member of the Advanced Broadband Communications Centre (CCABA) and of the Broadband Communications Research (CBA) group.

Josep Solè-Pareta received the M.Sc. degree in telecom engineering, in 1984 and the Ph.D. degree in computer science, in 1991, both from the Universitat Politècnica de Catalunya (UPC), Barcelona, Spain.

In 1984, he joined the Computer Architecture Department, UPC, where currently he is a Full Professor. He was a Postdoctoral Researcher between 1993 and 1994 summers at the Georgia Institute of Technology. He is cofounder of the UPC-CCABA. His current research interests are in autonomic communications, traffic monitoring and analysis, and high-speed and optical networking, with emphasis on traffic engineering, traffic characterization, MAC protocols, and QoS provisioning. He has authored or coauthored several book chapters and more than 120 papers in relevant research journals $(>25)$ and refereed interna- tional conferences. He has participated in many European projects dealing with computer networking topics.

Javier Aracil (S'94-SM'07) received the M.Sc. and Ph.D. degrees (with honors) from Universidad Politecnica de Madrid, Madrid, Spain, in 1993 and 1995, both in telecommunications engineering.

In 1995, he was appointed as a Postdoctoral Researcher at the Department of Electrical Engineering and Computer Sciences, University of California. In 1998, he worked as a Research Scholar at the Center for Advanced Telecommunications, Systems and Services, The University of Texas at Dallas. He has been an Associate Professor at University of Cantabria and Public University of Navarra, and he is currently a Full Professor at Universidad Autonoma de Madrid. His recent research interests include optical networks and performance evaluation of communication networks. He has authored or coauthored more than 100 papers in international conferences and journals.

Dr. Aracil was awarded with a Fulbright scholarship, in 1995. 